\title{
A FUNCTIONAL EQUATION FOR THE JOUKOWSKI TRANSFORMATION
}

\author{
MIROSLAW BARAN \\ (Communicated by Paul S. Muhly) \\ Dedicated to Ann $H$. on her 21st birthday
}

\begin{abstract}
The purpose of this paper is to solve a functional equation which arises from the Joukowski transformation. The functional equation has a simple geometric interpretation which is given.
\end{abstract}

\section{INTRODUCTION AND STATEMENT OF THE RESULTS}

In the paper [1, Proposition 2.1], it was observed that the transformation

$$
j(z)=\frac{1}{2}\left(z+z^{-1}\right) \quad(z \neq 0)
$$

satisfies the functional equation

$$
2 j(|z|)=|j(z)+1|+|j(z)-1| \quad \text { for } z \in D,
$$

where here and throughout $D:=\mathbf{C} \backslash\{0\}$. The transformation $j$ is said to be the Joukowski transformation and has applications in complex analysis and in aerodynamics. By (1) we get that the set $j(\{|z|=r\})$ is the ellipse with the great semiaxis equal to $j(r)$ and foci at -1 and +1 , if $r \neq 1$ or the line segment $[-1,1]$, if $r=1$. H. Haruki in his papers [2], [3] researched other equations arising from the Joukowski transformation.

The main result of this paper is the following theorem.

Theorem 1. Suppose that a function $f$ is holomorphic in $D$ and satisfies the functional equation

$$
2 f(|z|)=|f(z)+1|+|f(z)-1| \quad \text { for } z \in D .
$$

Then either $f=\mathrm{const} \geq 1$ or there exist $a>0, p \in \mathbf{N}$ such that

$$
f(z)=j\left((a z)^{p}\right) \quad \text { for } z \in D .
$$
that

$$
T_{n}(j(z))=j\left(z^{n}\right) \quad \text { for } z \in D, n \in \mathbf{N} .
$$

As a generalization of Theorem 1 we have the following:

Received by the editors August 1, 1988.

1980 Mathematics Subject Classification (1985 Revision). Primary 39B40; Secondary 33A70. 
Theorem 2. Fix $n \in \mathbf{N}$ and consider the equation

$$
2 T_{n} \circ f(|z|)=\left|T_{n} \circ f(z)+1\right|+\left|T_{n} \circ f(z)-1\right|, \quad z \in D .
$$

If $f$ is a holomorphic function in $D$ which satisfies (4) then either $f=$ const $=$ $C$, where $C$ has the form $C=\cos (2 \pi k / n)$ for some $0 \leq k \leq[n / 2]$ or $C=$ $j\left(\varepsilon C_{1}\right)$, where $C_{1} \geq 1, \varepsilon \in \mathbf{C}$ and $\varepsilon^{n}=1$, or there exist constants $a>0$, $p \in \mathbf{N}, \varepsilon \in \mathbf{C}, \varepsilon^{n}=1$ such that

$$
f(z)=j\left(\varepsilon(a z)^{p}\right) \text { for } z \in D .
$$

\section{Proof of Theorem}

If $f=$ const $=C$ then, by (2), must be $C \geq 1$. Assume that $f \neq$ const . Then $f(z)=\sum_{n=-\infty}^{+\infty} a_{n} z^{n}$, where the Laurent series is absolutely convergent for all $z \in D$ and $a_{n} \not \equiv 0$ for $n \neq 0$. If $r>0$ then, by (2), $f(r) \geq 1$ and $a_{n} \in \mathbf{R}$ for every $n \in \mathbf{Z}$. Hence $f(-r) \in \mathbf{R}$ for $r>0$. First we shall prove

Lemma 1. The function $f$ is even or is odd.

Proof. There exists $r_{0}>0$ such that $f\left(-r_{0}\right) \notin[-1,1]$, because, if $f(-r) \in$ $[-1,1]$ for all $r>0$ then we must have $f(r)=1, r>0$. By the identity property we have $f=$ const $=1$ and this is contrary to the assumption that $f \neq$ const. Since $f$ is continuous there exist $0<\alpha<r_{0}<\beta$ such that $f(-r) \notin[-1,1]$ for $\alpha<r<\beta$ and $|f(-r)|=f(r)$. This implies that $f(-r)=f(r)$ for $\alpha<r<\beta$ or $f(-r)=-f(r)$ for $\alpha<r<\beta$. By the identity property, $f(-z)=f(z)$ for $z \in D$ or $f(-z)=-f(z)$ for $z \in D$.

Next we prove an important fact.

Lemma 2. There exists $b>0$ such that $f(b)=1$.

Proof. The proof is by contradiction. Assume that $f(r)>1$ for all $r>0$. Let $\alpha:=\inf \{f(r): r>0\}, \beta:=\inf \{f(r): 0<r \leq 1\}, \gamma:=\inf \{f(r): r>1\}$. Consider two cases: (i) $\alpha>1$; (ii) $\alpha=1$. Let $f(z)=f_{1}(z)+f_{2}(z)$ where $f_{1}$ and $f_{2}$ are the singular part and the regular part of Laurent's expansion, respectively. The functions $f_{2}(z)$ and $f_{1}(1 / z)$ are entire. Suppose that $\alpha>1$. By (2) we have $|f(z)| \geq f(|z|)-1 \geq \alpha-1>0$. If $f_{1}(1 / z)$ and $f_{2}(z)$ are polynomials then the function $g(z)=z^{k} f(z), k=\operatorname{deg} f_{1}(1 / z)$, is a polynomial and $g(0) \neq 0,|g(z)| \geq|z|^{k}(\alpha-1)>0$ for $z \neq 0$. This means that $g=$ const $=C$ and $f(z)=C z^{-k}$, where $C=f(1)$. For $r=(f(1))^{1 / k}$ we have $f(r)=1$. If $f_{1}(1 / z)$ or $f_{2}(z)$ is a transcendental entire function then $z=0$ or $z=\infty$ is an essential singularity of $f$. By the Great Picard's Theorem (GPT), $\operatorname{card}(\mathbf{C} \backslash f(D)) \leq 1$. This is contrary to the statement that $|f(z)| \geq \alpha-1>0$. Assume that $\alpha=1$. If $\beta=\gamma=1$ then there exist sequences $r_{n} \searrow 0$ and $R_{n} \nearrow+\infty$ such that $f\left(r_{n}\right) \rightarrow 1$ and $f\left(R_{n}\right) \rightarrow 1$. Since $\lim _{n \rightarrow \infty} f_{2}\left(r_{n}\right)=$ $\lim _{n \rightarrow \infty} f_{1}\left(R_{n}\right)=0$ then there exists $M>0$ such that $\left|f_{1}\left(r_{n}\right)\right| \leq M$ and $\left|f_{2}\left(R_{n}\right)\right| \leq M$ for $n \in \mathbf{N}$. By (2) we have that the functions $f_{1}(1 / z)$ and $f_{2}(z)$ are bounded uniformly on the each circle $|z|=1 / r_{n}$ or $|z|=R_{n}$, respectively. 
By Liouville's theorem $f_{1}(z) \equiv 0$ and $f_{2}(z) \equiv a_{0}$ and $f(z) \equiv a_{0}$. This is also contrary to our general assumption. If $\beta=1$ and $\gamma>1$, then, as above, $f_{1}(z) \equiv 0$. Hence $f=f_{2}$ and $\left.f(0)=f_{2}(0)=1,|f(z)| \geq f\langle|z|)-1\right\rangle 0$ for $z \neq 0$. If $f$ is a polynomial then $f(z) \equiv f(1)$. The function $f$ can not be transcendental entire because $|f(z)| \geq \gamma-1\rangle 0$ for $|z| \geq 1$, what is contrary to GPT. In the case $\beta>1, \gamma=1$, due to Liouville's theorem and GPT, we also get a contradiction. The proof of Lemma 2 is complete.

Let $b>0$ and $f(b)=1$. Consider the function $g(z):=f(b z)$. The function $g$ is holomorphic in $D$, satisfies (2) and $g(1)=1$. Let $g(z)=$ $\sum_{n \in \mathbf{Z}} b_{n} z^{n}$, where $b_{n}=a_{n} b^{n}$. By (2) we get $g\left(e^{i \theta}\right) \in \mathbf{R}$ for every $\theta \in \mathbf{R}$. Thus we have $\sum_{n \in \mathbf{Z}} b_{n} e^{i n \theta}=\sum_{n \in \mathbf{Z}} b_{n} e^{-i n \theta}$ and we must have $b_{n}=b_{-n}$ for $n \in \mathbf{Z}$. Therefore $g(z)=b_{0}+\sum_{n \in \mathrm{N}} b_{n}\left(z^{n}+z^{-n}\right)$ for $z \in D$ and $g(z)=g(1 / z)$.

Lemma 3. There exists $p \in \mathbf{N}$ such that $g(z)=b_{0}+\sum_{n=1}^{p} b_{n}\left(z^{n}+z^{-n}\right)$ for $z \in D$.

Proof. Suppose that $z=0$ and $z=\infty$ are essential singularities of the function $g$. Let $h=\left.g\right|_{\mathbf{R}_{+}}, \alpha>1$ and $E=h^{-1}([1, \alpha])$. The set $E$ is closed in $\mathbf{R}_{+}$. If $E$ is compact then we have a contradiction to the GPT. If $E$ is unbounded then, due to Liouville's theorem, $g=$ const $=1$ (considerations in both cases are similar to those in the proof of Lemma 2). In every case we get a contradiction.

Finally we prove that $g(z)=\frac{1}{2}\left(z^{p}+z^{-p}\right)=j\left(z^{p}\right)$. By Lemma 1 the function $g$ is even or is odd. If $g$ is even then we can consider the function $g_{1}(z)=$ $g\left(z^{1 / 2}\right)$. The function $g_{1}$ is analytic for $z \in D$, satisfies (2) and $g_{1}(1)=1$. If $g_{1}$ is also even then we take $g_{2}(z)=g_{1}\left(z^{1 / 2}\right)$ and the function $g_{2}$ has the same properties as function $g_{1}$. If $g_{2}$ is even then we analogously define the function $g_{3}$. We can repeat this procedure. In the end we get the odd function $g_{k}$ such that $g(z)=g_{k}\left(z^{2^{k}}\right)$. Thus we can assume that $g$ is odd.

It is easy to verify that if the function $g$ satisfies (2) and $g(1)=1$ then the function $h(z)=2 g^{2}(z)-1$ also satisfies (2) and $h(1)=1$. The function $h$ is even and if $g$ is odd then the function $h\left(z^{1 / 2}\right)$ also must be odd. We have

$$
\begin{aligned}
h(z)= & 2 \sum_{\substack{n \leq p \\
n \equiv 1 \text { mod } 2}} b_{n}^{2}\left(z^{2 n}+z^{-2 n}\right)+4 \sum_{\substack{n<m \leq p \\
n, m=1 \bmod 2}} b_{n} b_{m}\left(\left(z^{n+m}+z^{-(n+m)}\right)\right. \\
& \left.+\left(z^{m-n}+z^{-(m-n)}\right)\right)+2 \sum_{\substack{n \leq p \\
n \equiv 1 \bmod 2}} b_{n}^{2}-1 .
\end{aligned}
$$

Thus we obtain

$$
\sum_{\substack{n<m \leq p \\ n, m=1 \bmod 2 \\ 4 \mid n+m}} b_{n} b_{m}\left(z^{n+m}+z^{-(n+m)}\right)+\sum_{\substack{n<m \leq p \\ n=1 \bmod 2 \\ 4 \mid m-n}} b_{n} b_{m}\left(z^{m-n}+z^{-(m-n)}\right) \equiv 0 .
$$


Let $p \equiv 1 \bmod 4$. Since $p-2 \equiv-1 \bmod 4$ and $n+m<2 p-2$ if $n<p-2$, we get that $b_{p-2}=0$. By induction we prove that $b_{p-2(2 k-1)}=0$ for $k=$ $1,2, \ldots,(p-1) / 4$. For $k=1$ we have $b_{p-2}=0$. Assume that $b_{p-2(2 k-1)}=0$ for $k=1, \ldots, l<\frac{p-1}{4}$ and consider the number $N=2 p-2(2 l+1)$. We have $N>p$ and if $n+m=N$ then we must have $n, m \geq p-2(2 l+1)$ and $0=\sum_{k=0}^{l+1} b_{p-4 k} b_{p-4 l-2+4 k}=b_{p} b_{p-4 l-2}$. Hence $b_{p-4 l-2}=0$. Because $4 \mid p-1$ and $m-n<p-1$ if $m<p$, we have $b_{1}=0$. By induction we can also prove that $b_{4 k-3}=0$ for $k=1, \ldots,(p-1) / 4$. If $p \equiv 3 \bmod 4$ then analogous considerations show that $b_{n}=0$ for $n \equiv 1 \bmod 2, n<p$.

Therefore we get $g(z)=a_{p}\left(z^{p}+z^{-p}\right)$ and since $g(1)=1$, we must have $g(z)=\frac{1}{2}\left(z^{p}+z^{-p}\right)$. If we take $a=1 / b$ then we obtain $f(z)=j\left((a z)^{p}\right)$. This completes the proof.

\section{Proof of Theorem 2}

By Theorem 1 we have two cases: (i) there exists $C_{1} \geq 1$ such that $T_{n}$ 。 $f(z) \equiv C_{1}$ or (ii) there exist $a>0, q \in \mathbf{N}$ such that $T_{n} \circ f(z)=j\left((a z)^{q}\right)$. Consider the first case. Since $T_{n}$ is a polynomial then the function $f$ must be equal to a constant $C$. If $C \in[-1,1]$ then, by the well-known properties of Chebyshev polynomials, we get that $C_{1}=1$ and $C=\cos (2 \pi k / n)$ for some $0 \leq k \leq[n / 2]$. Suppose that $C \notin[-1,1]$. Then we can write $C=j(h(C))$, where $h: \mathbf{C} \backslash[-1,1] \rightarrow \mathbf{C} \backslash \bar{B}(\bar{B}=\{|z| \leq 1\})$ is the inverse function to $j$, given by the formula $h(z)=z+\sqrt{z^{2}-1}$. Hence, by (3), $C_{1}=j\left(h^{n}(C)\right)$, $h^{n}(C)=h\left(C_{1}\right), h(C)=\varepsilon\left|h\left(C_{1}\right)\right|$ and finally $C=j\left(\varepsilon C_{2}\right)$ where $\varepsilon^{n}=1$ and $C_{2}=\left|h\left(C_{1}\right)\right|>1$. Now, assume that $f$ is not equal to a constant. As above, we have $f(z)=j(h(f(z)))$ for $z \in D_{1}=D \backslash f^{-1}([-1,1])$. Thus we obtain $j\left(h^{n}(f(z))\right)=j\left((a z)^{q}\right)$ for $z \in D_{1}, f^{-1}([-1,1]) \subset S(1 / a)$ and $h^{n}(f(z))=$ $(a z)^{q}$ for $z \in D_{2}=D_{1} \cap(C \backslash \bar{B}(1 / a))$, where $\bar{B}(1 / a), S(1 / a)$ denote the ball and the circle with centre at zero and radius $1 / a$. Since the domain $D_{3}=$ $D_{2} \backslash(-\infty, 0)$ is simply connected, then there exists in $D_{3}$ the principal branch of the function $(a z)^{q / n}$, which we will also denote by $(a z)^{q / n}$. Next, we can write $h^{n}(f(z))=\left((a z)^{q / n}\right)^{n}$ and we deduce that there exists $\varepsilon$, an $n$-th root of 1 , such that $h(f(z)))=\varepsilon(a z)^{q / n}$ for $z \in D_{3}$ and $f(z)=j\left(\varepsilon(a z)^{q / n}\right.$ ) for $z \in D_{3}$. Using analytic continuation we extend this equality to the domain $D \backslash(-\infty, 0)$. Finally, observe that since $f$ is holomorphic in $D$, then we must have $q \mid n$. If we put $p=q / n$ then we complete the proof.

\section{REFERENCES}

1. M. Baran, Siciak's extremal function for convex sets in $\mathbf{C}^{N}$, Annales Polon. Math. 48 (1988), in press.

2. H. Haruki, A functional equation arising from the Joukowski transformation, Annales Polon. Math. 45 (1985), 185-191.

3. __ A new functional equation characterising generalized Joukowski transformations, Aequationes Math. 32 (1987), 327-335.

Department of Mathematics, Pedagogical University of Cracow, Cracow, Poland 